\title{
Mnemonic and Histopathological Assessment of the Neuroprotective Effects of Murraya Koenigii Leaves Extract in Rats with Partial Global Cerebral Ischaemia
}

\author{
Marwan Saad Azzubaidi ${ }^{a}$, Imad M. Al-Ani ${ }^{b}$ \\ aPharmacology Unit, Faculty of Medicine, Universiti Sultan Zainal-Abidin, Kuala Terengganu, Malaysia \\ ${ }^{b}$ Basic Medical Sciences Department, Faculty of Medicine, International Islamic University Malaysia, \\ Kuantan campus
}

\begin{abstract}
Introduction: Preclinical studies have reported that Murraya koenigii leaves (MKL) could enhance memory. MKL is also known for its antioxidant activity. The current study was to assess the possible neuroprotective potential of MKL methanolic extract in a two vessel occlusion (2VO) rat model of partial global cerebral ischaemia. Methods: Rats were divided into memory and learning groups. Each group was subdivided into sham control, untreated $2 \mathrm{VO}$ and MKL-treated $2 \mathrm{VO}$ subgroups. The Morris water maze test was implemented to assess the rats' cognitive function postoperatively. Brain samples were histopathologically examined for viable neurons within the CA1 hippocampal region. Results: Water maze test findings showed that MKL positively improved memory and learning impairments. However, this improvement in memory test for the treated group was still significantly inferior to that of the healthy control group. Additionally, MKL treated group exhibited insignificant difference in the number of viable hippocampal CA1 pyramidal neurons from that of the untreated 2VO group, whereas both MKL treated and untreated 2VO groups showed significantly less viable neurons when compared with the control group. Conclusion: MKL extract modestly improved memory without providing substantial neuroprotective action to the hippocampal neurons in rats with chronic partial global cerebral ischaemia.
\end{abstract}

KEYWORDS: Murraya Koenigii, Spatial memory, Neuroprotection, Cerebral hypoperfusion, neurodegeneration, Pyramidal cell, Hippocampus

\section{INTRODUCTION}

Murraya Koenigii leaves (MKL), belonging to the plant family "Rutaceae", generally known as curry patta, is a popular Indian food spice and a wellknown Ayurvedic medicinal plant. Traditionally, MKL is utilized to treat vomiting, diarrhoea and also used as a general tonic or an energy boosting agent. ${ }^{1}$ Evidence based reports within the last decade highlighted its robust antioxidant, ${ }^{2}$ and reactive

Corresponding Author:

Assoc. Prof. Dr. Marwan Saad Azzubaidi,

Faculty of Medicine,

University Sultan Zainal Abidin, Campus Kota,

Kuala Terengganu, Malaysia,

Tel No: +6 0189066351

Email: mazzubaidi@gmail.com nitrogen species scavenging activities. ${ }^{3}$ These potentially beneficial mechanisms were attributed to some carbazole alkaloids that were identified as predominant active ingredients of MKL. ${ }^{4}$

Recently, two important studies on experimental animals with drug induced amnesia asserted that MKL and its ethanolic extract could significantly enhance memory and prevent scopolamine triggered memory impairment. Both studies have related this mnemonic activity to acetylcholine release stimulation and/or anticholinesterase mechanism of MKL action with a final recommendation for future research to test the neuroprotective effects of the MKL extract on a more scientific animal model of Alzheimer's disease (AD). ${ }^{5,6}$ Permanent two vessel occlusion (2VO) surgery is now broadly employed to induce hippocampal neurodegeneration as a result of global long standing cerebrovascular hypoperfusion. 
The resultant hippocampal neuronal cell loss in 2VO rats is said to be closely resembling that of $A D$ both in its origin and outcome. As for its origin, it is now widely accepted that sporadic $A D$ may result from one or more vascular risk factors that lead to chronic cerebral hypoperfusion which in turn would impede the transport of essential nutrients to the brain. ${ }^{8}$ Concerning their outcomes, both $A D$ patients and $2 \mathrm{VO}$ rats were found to suffer progressive spatial memory decline as an early manifestation of hippocampal neurodegeneration. ${ }^{9}$ Moreover, oxidative stress and neuroinflammation are thought to play a pivotal role in $A D$ pathogenesis ${ }^{10}$ as well as in $2 \mathrm{VO}$ induced neurodegeneration in rats. ${ }^{11}$ Since the CA1 hippocampal region was reported to be the most sensitive area for neurodegeneration following $2 \mathrm{VO}$ intervention, ${ }^{12}$ the current study was designed to assess the potential neuroprotective effects of the MKL extract on chronically hypoperfused rat's brain that resulted from $2 \mathrm{VO}$ intervention. The assessment was based on learning and memory test protocols, using the Morris water maze (MWM) apparatus, and histopathological examination of hippocampal sections.

\section{METHODS}

\section{MKL extraction}

Fresh curry leaves $(10 \mathrm{~kg})$ were harvested from Pandan River Forest, a few kilometres away from Kuantan. MKL was identified and authenticated by a taxonomist in the Faculty of Pharmacy, IIUM. Voucher specimen was deposited at the Faculty's herbarium. The leaves were left to dry before they were gounded to powder and dissolved in methanol under magnetic stirrer for 24 hours. The pure extract was separated on the next day using Rotary Evaporator apparatus. The total yield of MKL extract was 200 grams.

\section{Animal groups}

Sixty male Sprague Dawley rats were purchased from the Faculty of Veterinary Medicine, University Putra Malaysia. The rats' weights range was 250-350 grams on the date of surgery. Animals had free access to water and food pellets throughout the study and an equal daily exposure to light and darkness. Rats were treated according to the guidelines for the care and use of mammals in neuroscience and behavioural research as well as the recommendations of the IIUM ethical committee. After an acclimatization period of 7 days, animals were randomly and equally divided into 3 main groups: Goup A (vehicle treated and sham operated), labelled as sham. Group B (vehicle treated and 2VO operated), labelled as 2VO. Group C (MKL+vehicle treated and 2VO operated), labelled as MKL.

The daily oral dose of $M K L(50 \mathrm{mg} / \mathrm{kg})$ was commenced 10 days before the date of $2 \mathrm{VO}$ surgery and continued until the end of the $10^{\text {th }}$ postoperative week (day70) as shown in Fig.1.

Each one of the main groups was further subdivided into 2 subgroups. Long-term memory (LTM) subgroups and short-term memory + working memory (STM+WM) subgroups (Figure1A).

\section{$2 \mathrm{VO}$ surgery}

Under combined intraperitoneal general anaesthesia consisting of ketamine $(90 \mathrm{mg} / \mathrm{kg})$ and xylazine $(10 \mathrm{mg} / \mathrm{kg})$, the common carotid arteries were bilaterally exposed, separated from the vagus nerve, permanently double ligated with a silk suture and cut in between the two ligatures. Rats were kept under a heating lamp until full recovery from anaesthesia. Postoperative buprenorphine was given intramuscularly to selected animals which developed signs of distress or pain.

\section{MWM apparatus}

A black fibre glass circular tank, 2 meter in diameter, was used for evaluating LTM, STM and working memory (WM). A black platform, $10 \mathrm{~cm}$ in diameter, was submerged below the water throughout all test protocols, except during habituation training before the first test day and during the single cued version trial at the end of the last test trial.

In LTM test protocol, the animals were trained on the swimming Task before undergoing $2 \mathrm{VO}$ surgery. The position of the platform remained fixed (in the left lower quadrant) for 3 consecutive days with 4 trials per day with a different starting point of each trial. The maximum duration of time allowed for each animal during a trial was 120 seconds. 


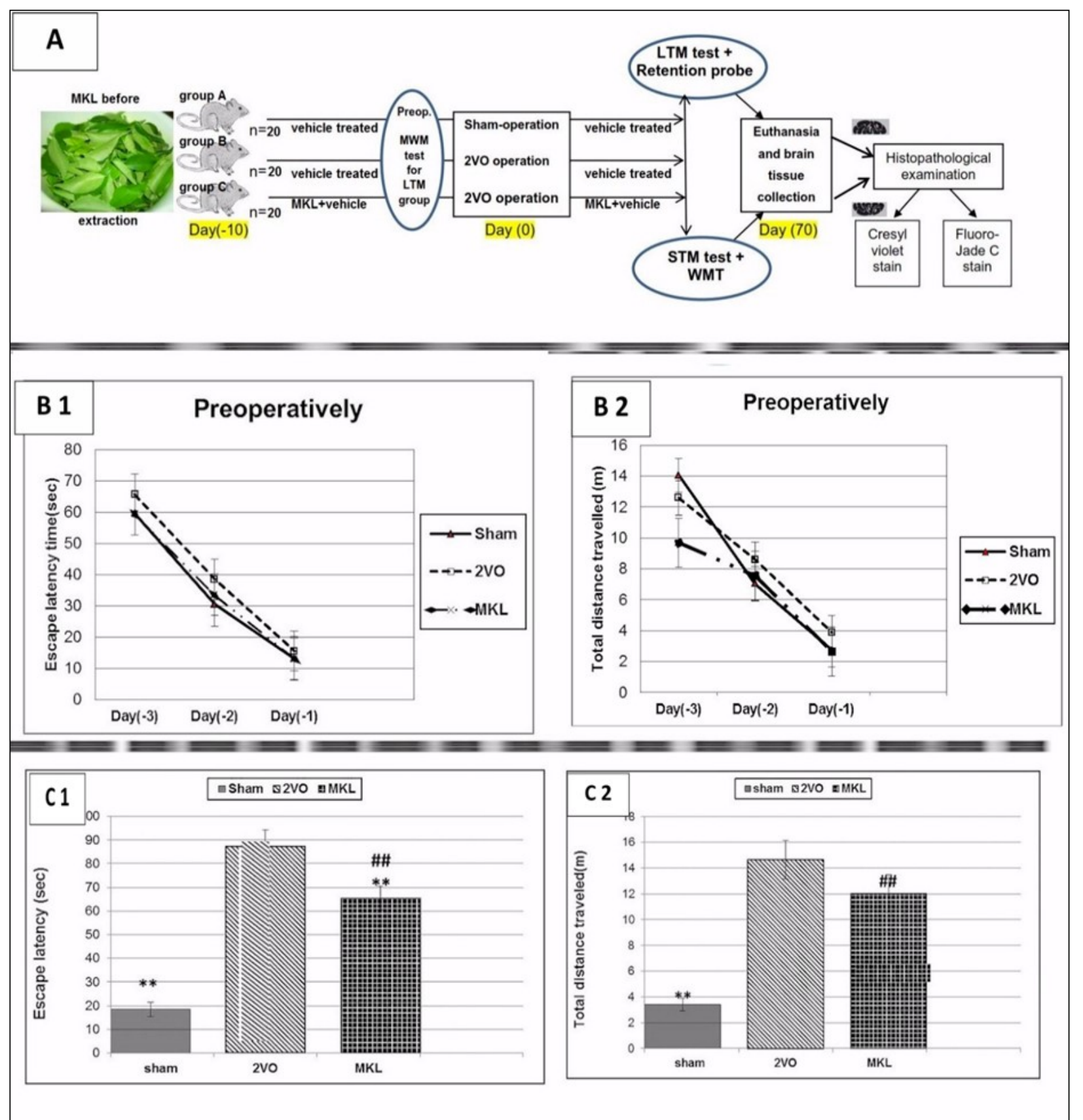

Figure 1. (A): Experimental protocols for the study groups. LTM= Long-term memory, STM= short-term memory, WMT= working memory test. (B1\& B2): LTM acquisition trials depicting improved daily performance of all groups in (B1) escape latency (B2) pathlength without any significant differences among groups. (C1\& C2): LTM test depicting significantly shorter results of the sham group as compared to $2 \mathrm{VO}$ and MKL groups in (C1) escape latency (C2) pathlength. ${ }^{* *} \mathrm{p}<0.01$ as compared to $2 \mathrm{VO}$, \#\# $\mathrm{p}<0.01$ as compared to sham.

Animals which failed to locate the platform within the 120 seconds were gently guided to the platform and left there for 30 seconds, so as to learn its position in relation to the extra-maze visual cues (coloured posters pasted to the lab walls). Parameters recorded for each animal by Anymaze video tracking software (Stoelting Co., USA) were escape latency time, path-length and swimming speed. At the tenth week post $2 \mathrm{VO}$ all animals of LTM group were subjected again to a single day test consisting of 4 trials. This was followed on the next day by retention probe single trial memory test for
60 seconds with the platform removed from the pool. During the probe test, the parameters recorded were the time spent by each animal in the target (left lower) zone and the total number of annulus crossings.

In STM test protocol the animals were subjected for the first time to MWM on the $10^{\text {th }}$ postoperative week (no preoperative training). The test protocol was for 4 consecutive days followed on the fifth day of the retention probe memory test with similar parameters recorded as those with LTM protocol where the 
platform was constantly hidden below the water surface in the same left lower quadrant. WMT was conducted for 3 successive days after the last probe memory test for STM subgroups. In WMT protocol the platform's location was changed on a daily basis among the right upper, right lower and left upper quadrants of the pool as described in table 1. Parameters recorded were the escape latency time, total pathlength until reaching the platform and swimming speed.

Table 1: MWM protocols for LTM, STM and WM

\begin{tabular}{lccc}
\hline & LTM & STM & $\begin{array}{c}\text { WMT } \\
\text { (learning) }\end{array}$ \\
\hline $\begin{array}{l}\text { Preoperative MWM } \\
\text { training }\end{array}$ & Yes & No & No \\
$\begin{array}{l}\text { Acquisition trials } \\
\text { Daily alteration of } \\
\text { platform position } \\
\text { inside the pool } \\
\begin{array}{l}\text { Retention probe } \\
\text { memory test }\end{array}\end{array}$ & Yes & Yes & No \\
\hline
\end{tabular}

subgroups.

Histopathology

At the end of the tenth postoperative week all animals were humanely euthanized using an overdose of inhaled diethyl ether. The brains were rapidly collected to be fixed in 10\% formaldehyde for $72 \mathrm{hrs}$. Coronal hippocampal sections were prepared from the right and the left hemispheres to be stained with cresyl violet and FJC stains, respectively. Staining procedures were similar to those described by previous studies. ${ }^{7,13}$ Slides were examined by a histopathologist who was blinded to the study groups. Calculation of viable pyramidal neurons within $1 \mathrm{~mm}^{2}$ of the CA1 hippocampal subfield was performed according to the method described previously by Azzubaidi and co-workers. ${ }^{14}$

\section{Statistical analysis}

Anymaze software analysed differences in mean values of escape latencies, pathlengths and swimming speeds using two way ANOVA with one repeated measure (both factors fixed). Data from retention probe memory tests and the mean viable neurons of the study groups were subjected to a one way ANOVA with post hoc Tukey's test for groups' comparisons using SPSS software version 17.0. Results were expressed as mean (SEM). $p$ values less than 0.05 were considered statistically significant.

\section{RESULTS}

\section{Preoperative acquisition trials}

No significant differences among groups were observed during the pre $2 \mathrm{VO}$ acquisition trials of LTM test, neither in escape latency $[F(2,117)=0.88, p=$ 0.41] (Figure1 B1) nor in the pathlengths until reaching the platform $[F(2,117)=2.85, \quad p=0.06]$ (Figure1 B2).

\section{Postoperative Mortality and Blindness rates}

Table 2 illustrates post 2VO mortality and blindness rates. Blind rats were excluded from the MWM test as it requires satisfactory vision for the animal to build its spatial memory.

Table 2: Postoperative mortality and blindness rates among study groups.

\begin{tabular}{cccc}
\hline Postoperative rates & sham & 2VO & MKL \\
\hline Mortality & $(0 \%)$ & $(42.10 \%)^{* *}$ & $(30 \%)^{* *}$ \\
Blindness & $(0 \%)$ & $(9 \%)$ & $(5 \%)$ \\
Total operated & 20 & 38 & 30 \\
\hline
\end{tabular}

${ }^{* *} p<0.01$ versus sham group.

\section{LTM results}

Escape latency results of the tenth postoperative week showed significantly shorter time required by the sham group in comparison with the other 2 groups $[F(3,35)=30.43, p<0.01]$. The escape latency time of $\mathrm{MKL}$ group also was significantly shorter than that of $2 \mathrm{VO}$ group ( $p<0.01$ ) (Figure1 C1). Whereas the pathlength results showed that the difference was significant only when comparing sham with the other 2 groups [F $(3,35)=18.12, p$ $<0.01]$. That is to say, the total distance travelled by MKL and 2 VO were not statistically significant $(p=$ 0.06) (Figure1 C2).

\section{Retention probe LTM results}

The sham group demonstrated significantly longer time in the target zone of the maze than $2 \mathrm{VO}$ and MKL groups $[F(3,33)=9.14, p<0.01]$. However, difference between $2 \mathrm{VO}$ and $\mathrm{MKL}$ groups was not significant ( $p=0.10$ ) (Figure $2 A 1)$. In comparing the number of annulus crossings among all groups no significant difference was found $[F(3,33)=2.33, p=$ 0.09] (Figure $2 \mathrm{~A} 2$ ). 


\section{STM Acquisition results}

Both sham and MKL groups showed significantly shorter escape latency time $[F(2,27)=34.49, p<$ 0.01] (Figure $2 \mathrm{~B} 1$ ) and pathlengths travelled [F $(3,36)=3.91, p=0.01$ ] (Figure $2 \mathrm{~B} 2$ ) than that of VO group. Nevertheless, the difference was found to be significant between sham and MKL groups in both parameters.

\section{Retention probe STM results}

The sham group demonstrated significantly longer time spent in the target zone of the maze $[F(3,36)$ $=20.79, \mathrm{p}<0.01]$ than $2 \mathrm{VO}$ and MKL groups. Yet, $M K L$ group also showed significantly longer duration $(p<0.01)$ as compared to 2VO group (Figure $2 \mathrm{C} 1$ ). Sham group also showed a significantly higher number of annulus crossings than both $2 \mathrm{VO}$ and $M K L$ groups $[F(3,36)=6.56, p<0.01]$, while the difference between $2 \mathrm{VO}$ and MKL groups was not significant $(p=0.29)$ (Figure $2 \mathrm{C2}$ ).

\section{WMT results}

There were significant differences among all groups, both in escape latency $[F(3,36)=38.11, p$
$<0.01]$ and pathlength $[F(3,36)=37.06, p<0.01]$ on each of the 3 WMT days (Figure $3 A 1 \& A 2$ ).

\section{Swimming speed results}

Both LTM and (STM+WM) subgroups showed no significant differences in swimming speed during the last phase of the test $[F(3,35)=0.06, p=0.9]$ and $[F$ $(2,27)=0.07, p=0.9]$, respectively (Figure $3 \mathrm{~B} 1 \&$ B2).

\section{Histopathology results}

\section{A-FJC stain results}

Under a fluorescent microscope with FITC filter the three groups did not show any difference in their fluorescence intensity within CA1 pyramidal hippocampal layer (Figure 3 C1- C3).

\section{B-Cresyl violet stain}

\section{1- Qualitative assessment}

Pyramidal neurons having regular boundaries, a light colored cytoplasm and a distinct nucleus were considered normal and counted as viable (Figure 4 A), whereas shrunken cells that lost their characteristic triangular outlines with irregular cell membranes and darkly stained cytoplasm

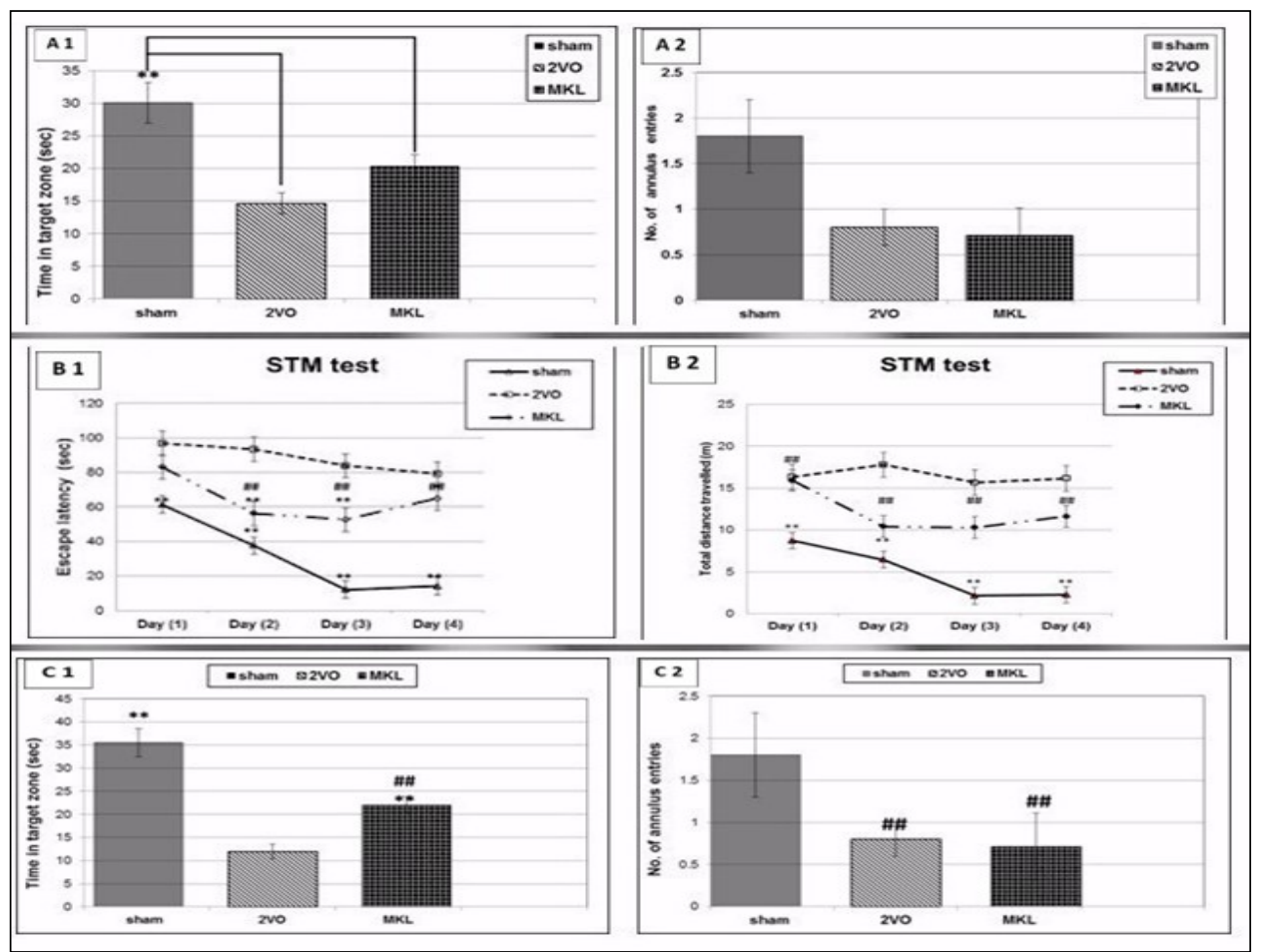

Figure 2. (A1 \& A2): Retention probe LMT is depicting differences among groups in (A1) time spent in the target (left lower) zone (A2) number of annulus crossings. ${ }^{*} P<0.01$ as compared to the other 2 groups. (B1 \& B2): STM result is depicted significantly shorter time (B1) and distance travelled (B2) for sham and MKL groups than those of $2 \mathrm{VO}$ group. ${ }^{* *} \mathrm{p}<0.01$ as compared to $2 \mathrm{VO}, \# \# \mathrm{p}<0.01$ as compared to sham. (C1 \& C2): Retention probe SMT is depicting differences among groups in (C1) time spent in the target (left lower) zone (C2) number of annulus crossings. ${ }^{* *} \mathrm{p}<0.01$ as compared to $2 \mathrm{VO}, \# \# \mathrm{p}<0.01$ as compared to sham. 


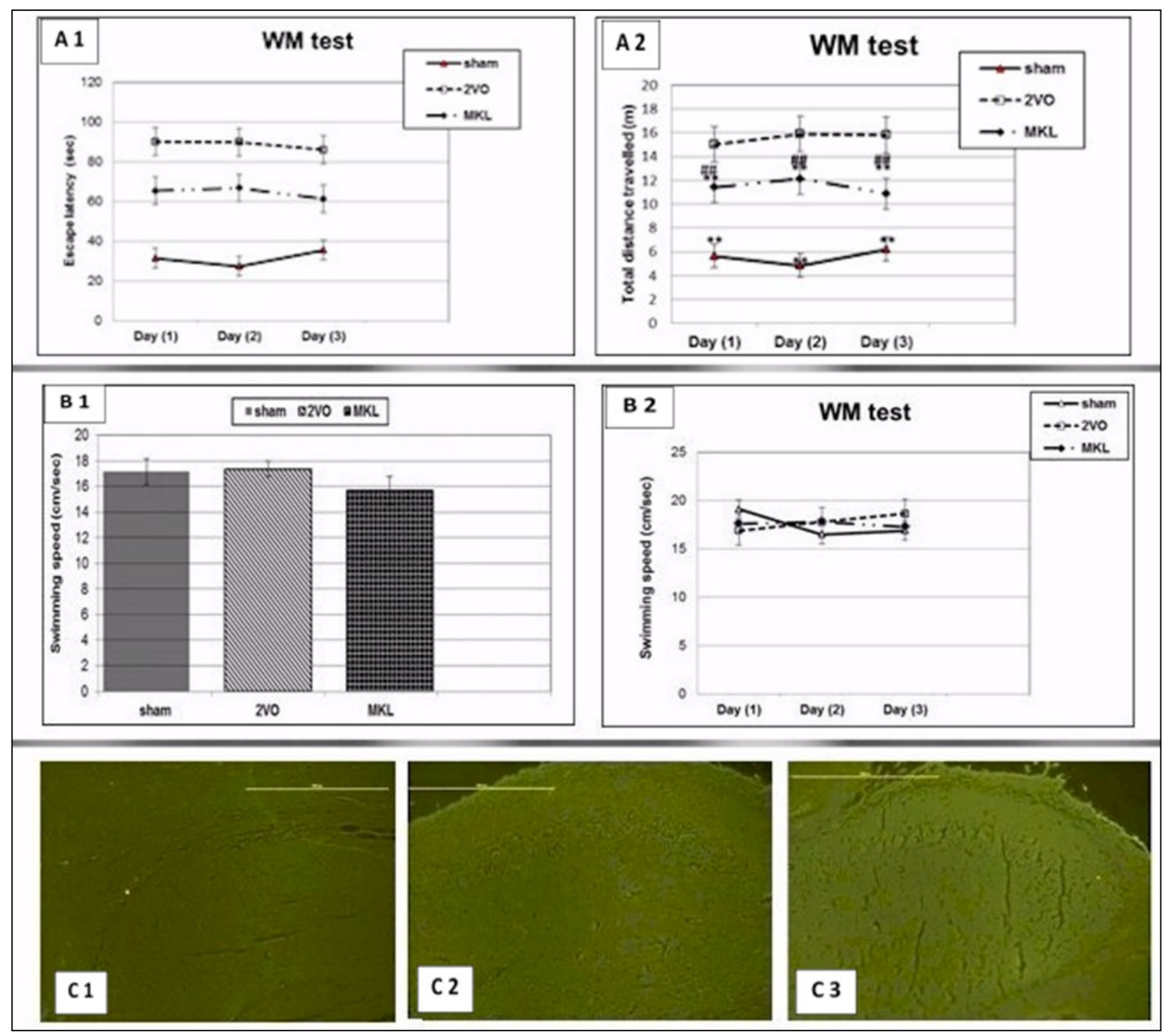

Figure 3. (A1\&A2): WMT result is depicted significantly longer (A1) duration and (A2 ) distance travelled by 2 VO and MKL groups as compared to the sham group. ${ }^{* *} \mathrm{p}<0.01$ as compared to $2 \mathrm{VO}, \# \# \mathrm{p}<0.01$ as compared to sham. (B1\&B2): Swimming speed results from (B1) LTM test (B2) WMT depicting insignificant differences among tested groups. (C1, C2 \&C3): Light photomicrograph depicting CA1 pyramidal cells with no significant difference between study groups: sham (C1), $2 \mathrm{VO}(\mathrm{C2})$ and MKL (C3). Scale bar $=500 \mu \mathrm{m}$.

indistinguishable from the nucleus were considered degenerated and therefore not counted as viable in $2 \mathrm{VO}$ treated rats (Figure $4 \mathrm{~B}$ ). Treatment of $2 \mathrm{VO}$ rats with MKL (group C) showed normal pyramidal cells dominated treated rat hippocampal sections with clear and well defined nucleus (Figure 4C).

\section{2- Quantitative assessment}

The mean viable CA1 pyramidal neurons were significantly higher $(p<0.01)$ in sham group than both $2 \mathrm{VO}$ and MKL groups. However, the difference was insignificant $(p=0.37)$ when comparing MKL with $2 \mathrm{VO}$ viable neuronal count (Figure $4 \mathrm{D}$ ).

\section{DISCUSSION}

The promising preliminary results of MKL extract as an antioxidant, ${ }^{2}$ and memory enhancing agent 5,15 prompted researchers to conduct a more specific study to test the neuroprotective potential of this medicinal plant. The permanent $2 \mathrm{VO}$ rat model is gaining more acceptance worldwide as a paradigm exemplifying the vascular hypothesis for $A D,{ }^{16,17}$ MKL extract was tested in this $2 \mathrm{VO}$ model with the aim of evaluating its expected neuroprotective properties before the neurodegenerative process takes place. For that reason MKL extract was administered 10 days prior to the date of inducing cerebrovascular hypoperfusion. This was of particular importance because of the general concept that the resulting neurodegeneration in $A D$ as well as in $2 \mathrm{VO}$ rat model is irreversible though preventable. ${ }^{16}$

Significant early postoperative mortality rate may reflect a primary evidence that MKL pre-treatment could not protect the vital centers of the brain from the immediate diminishment in metabolic supplies which resulted from the sudden acute drop in cerebral blood flow. This postoperative mortality 


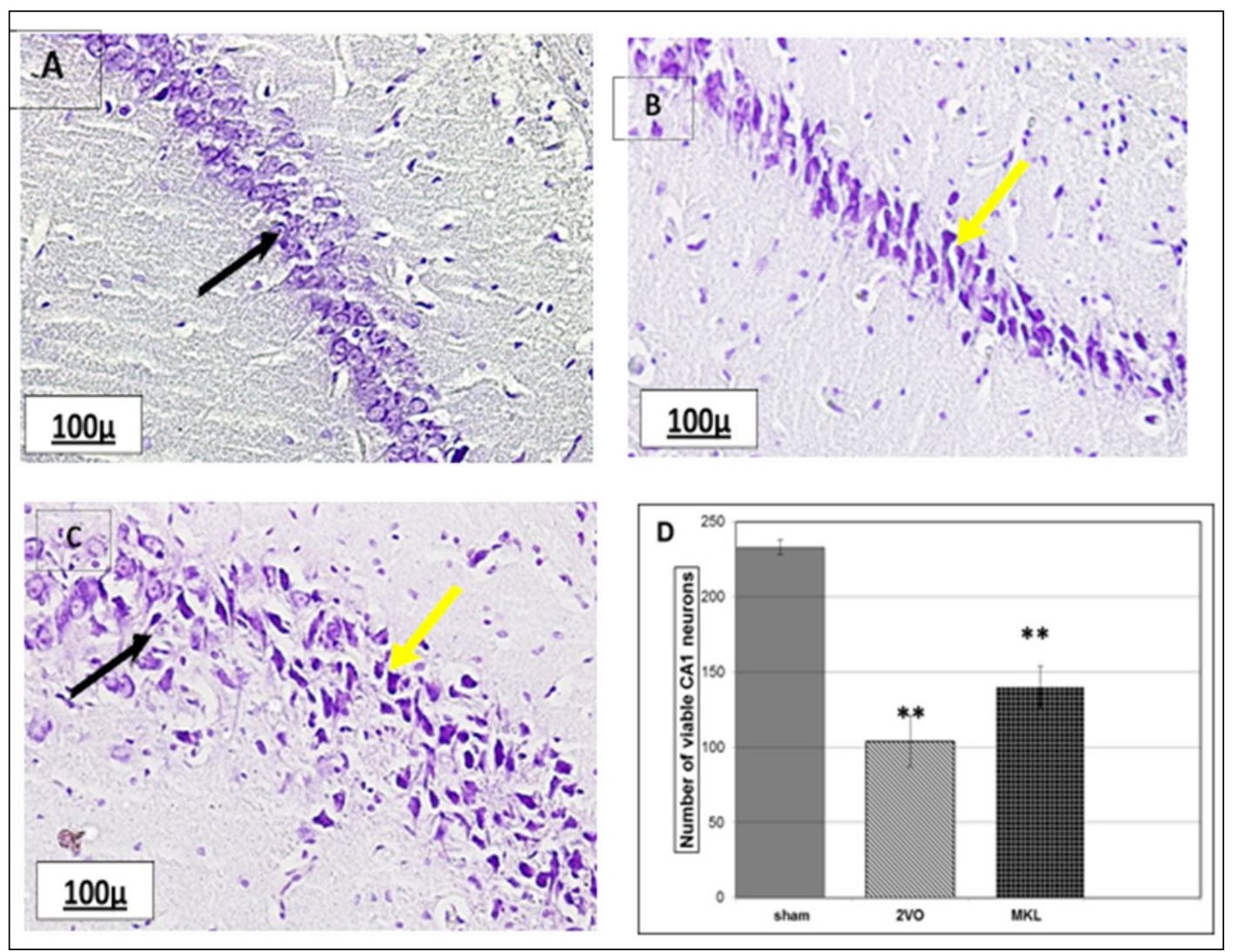

Figure 4. Cresyl violet stained light photomicrographs depicting CA1 pyramidal neurons from sham group (A), 2Vo group (B), MKL group (C) and a quantitative comparison of the viable pyramidal neuron of the 3 study groups (D). Black arrows $=$ viable pyramidal cells, yellow arrows $=$ degenerated pyramidal cells. ${ }^{* *} \mathrm{p}<0.01$ versus sham group.

was found to be of a much lower rate in rats treated with other medicinal plant extracts, ${ }^{18}$ which might indicate a lower efficacy of MKL neuroprotective potential. Significant impairment in the water maze performance of all untreated 2VO subgroups in LTM, STM and WM protocols 10 weeks post $2 \mathrm{VO}$ surgery as compared to the sham group denotes severe deterioration in these cognitive functions. This is in agreement with the results of other studies. ${ }^{19-21}$

MKL treated group did not show a consistent performance throughout the MWM tests. The significantly shorter escape latency time of MKL treated than $2 \mathrm{VO}$ group was simultaneously significantly longer (worse) than that of the sham control group. Moreover, the comparable durations of time spent in the target zone for untreated $2 \mathrm{VO}$ and MKL treated groups that were both significantly shorter than the sham group during the retention probe memory test is another indication of MKL modest preventive action against LTM impairment induced by $2 \mathrm{VO}$. This was somehow contrast to what was reported by other researchers who asserted a significant improvement in memory and learning for
MKL treated group. ${ }^{5,6}$ The dissimilarity in our findings as compared to the aforementioned studies may be ascribed, on one hand, to differences for modelling memory impairment, and to the different procedures employed to assess memory and learning performances among tested groups on the other hand. While the method adopted in the above studies to establish the memory impairment were drug induced amnesia and by using aged mice, 2VO is considered a more specific model for neurodegeneration giving a clearer picture about the expected neuroprotection of the tested agent. Additionally, the chronic nature of our study (animals tested 10 weeks postoperatively) may provide a better and more accurate assessment of the probable neuroprotection and memory enhancement than in other shorter studies. The chronicity of the designed study is essential since the exhaustion of intracellular stores of neurotransmitters involved in memory and learning is time dependent. ${ }^{22}$ As for the procedures used to evaluate memory performance, MWM test is considered superior to other tests, including radial arm and elevated plus mazes in assessing LTM, STM 
and WM because of lower possibility of test biases, more reliable tendency to finish the swimming task by the rats and fewer chances of developing olfactory cues for MWM in comparison with other behavioural tests. ${ }^{23}$ Absence of significant swimming speed differences throughout LTM and WM tests (results not shown) reassured that the tested parameters were not confounded by differences in swimming speed. Since it has already been reported that MKL treatment inhibits cholinesterase enzyme and thereby increasing acetylcholine levels in the brain, ${ }^{6}$ there is no doubt that the good water maze rats performances during STM and WM tests in comparison with the untreated group was due to sustainable quantities of neurotransmitters that were able to maintain the memory and learning faculty of the treated group at a significantly higher level than that of the untreated $2 \mathrm{VO}$ group. The question, however, remained of whether this significant difference was merely a result of acetylcholinesterase enzyme inhibition or due to tangible neuroprotective activity exerted by the carbazole alkaloid compounds of MKL which are thought to possess potent antioxidant actions. ${ }^{15}$

Quantitative comparison of viable pyramidal cell numbers among the three groups clearly showed that there was an insubstantial neuroprotective action from MKL treatment, at least within the CA1 hippocampal subfield, as the mean viable neuronal cell count was significantly lower in the treated group than that of sham group. The negative findings of FJC stain was against our prediction since it is regarded as highly specific for detecting neurodegenerated cells and even axons under fluorescent microscope. ${ }^{13}$

Lack of high fluorescent intensity within the untreated 2VO group was in contrast to Ehara and Ewidi who to induce cerebral neurodegeneration by chemical intoxication of the brain and observed FJC-positive fibres, but little in cell bodies of the neurons in the substantia nigra of the male homozygous zitter mutant (zi/zi) rats. ${ }^{24}$ Nevertheless, our negative FJC findings in untreated $2 \mathrm{VO}$ group stood in agreement with the observation of Ritchie et al.; who stained the hippocampi of Sprague-Dawley rats with the same fluorescent stain 6 weeks post $2 \mathrm{VO}$ and postulated that this negative finding may indicate that the neurodegenerative event might have happened at an earlier stage that rendered it impossible to be picked up by the fluorescent stain at the later stage; ${ }^{25}$ it might be related to species differences.

\section{CONCLUSION}

Our study concludes that the memory enhancing activity achieved by MKL treatment cannot be attributed to a tangible neuroprotective action, but rather to increase acetylcholine levels in the brain secondary to acetylcholinesterase enzyme inhibition.

\section{CONFLICT OF INTEREST}

Authors declare that there are no competing interests of any kind.

\section{ACKNOWLEDGEMENT}

The authors would like to thank IIUM research management center for sponsoring this study under a research grant number (EDW B 11-215-0693). The authors would also like to show their appreciation to Associate Professor Dr. Qamar U. Ahmed for directing medicinal plant extraction.

\section{REFERNCES}

1. Medina LS \& Zurakowski D. Measurement Variability and Confidence Intervals in Medicine: Why Should Radiologists Care? Radiology 2003; 226: https://doi.org/10.1148/ radiol.2262011537

2. Tachibana $\mathrm{Y}$, Kikuzaki $\mathrm{H}$, Lajis $\mathrm{NH}$, et al. Antioxidative activity of carbazoles from Murraya koenigii leaves. Journal of agricultural and food chemistry 2001; 49: 5589-94.

3. Baliga MS, Jagetia GC, Rao SK, Babu SK. Evaluation of nitric oxide scavenging activity of certain spices in vitro: A preliminary study. Food/Nahrung 2003; 47: 261-4.

4. Adebajo A, Ayoola O, Iwalewa E, et al. Antitrichomonal, biochemical and toxicological activities of methanolic extract and some carbazole alkaloids isolated from the leaves of Murraya koenigii growing in Nigeria. Phytomedicine 2006; 13: 246-54.

5. Vasudevan M, Parle M. Antiamnesic potential of Murraya koenigii leaves. Phytotherapy Research 2009; 23: 308-16.

6. Tembhurne S, Sakarkar D. Beneficial effects of ethanolic extract of Murraya koenigii (Linn) 
leaves in cognitive deficit aged mice involving possible anticholinesterase and cholesterol lowering mechanism. Int J PharmTech Res 2010; 2: 181-8.

7. Farkas E, Luiten PGM, Bari F. Permanent, bilateral common carotid artery occlusion in the rat: a model for chronic cerebral hypoperfusion-related neurodegenerative diseases. Brain Research Reviews 2007; 54: 162-80.

8. de la Torre JC. Vascular risk factor detection and control may prevent Alzheimer's disease. Ageing Res Rev 2010; 9: 218-25.

9. de la Torre JC. Alzheimer's disease is incurable but preventable. Journal of Alzheimer's Disease 2010; 20: 861-70.

10. Bamberger ME, Landreth GE. Inflammation, apoptosis, and Alzheimer's disease. The Neuroscientist 2002; 8: 276-83.

11. Van Dam D, Coen K, De Deyn PP. Ibuprofen modifies cognitive disease progression in an Alzheimer's mouse model. Journal of Psychopharmacology 2010; 24: 383-8.

12. Marosi M, Fuzik J, Nagy D, et al. Oxaloacetate restores the long-term potentiation impaired in rat hippocampus CA1 region by 2-vessel occlusion. European Journal of Pharmacology 2009; 604: 51-7.

13. Schmued LC, Stowers CC, Scallet AC, XU L. Fluoro-Jade $C$ results in ultra-high resolution and contrast labelling of degenerating neurons. Brain Research 2005; 1035: 24-31.

14. Azzubaidi MS, Saxena AK, Talib NA. Quantifying dorsal Hippocampal CA-1 pyramidal cells in rats: rules to light microscope based estimation. Malaysian Journal of Microscopy 2013; 9, 165-9

15. Mani V, Ramasamy K, Ahmad A, et al. Effects of the Total Alkaloidal Extract of Murraya koenigii Leaf on Oxidative Stress and Cholinergic Transmission in Aged Mice. Phytotherapy Research 2013; 27: 46 - 53.

16. de la Torre JC. The vascular hypothesis of Alzheimer's disease: bench to bedside and beyond. Neuro-degenerative diseases 2010; 7: 116-21.

17. Obrenovich ME, Smith MA, Siedlak SL, et al. Overexpression of GRK2 in Alzheimer disease and in a chronic hypoperfusion rat model is an early marker of brain mitochondrial lesions. Neurotoxicity Research 2006; 10: 43 56.
18. Azzubaidi MS, Saxena AK, Talib NA, et al. Protective effect of treatment with black cumin oil on spatial cognitive functions of rats that suffered global cerebrovascular hypoperfusion. Acta neurobiologiae experimentalis 2012; 72: 154-65.

19. de la Torre JC, Aliev G. Inhibition of vascular nitric oxide after rat chronic brain hypoperfusion: spatial memory and immunocytochemical changes. Journal of cerebral blood flow and metabolism 2005; 25 : 663-72.

20. Ji HJ, Hu JF, Wang YH, et al. Osthole improves chronic cerebral hypoperfusion induced cognitive deficits and neuronal damage in hippocampus. European Journal of Pharmacology 2010; 636: 96-101.

21. Liu HX, Zhang JJ, Zheng $P$, et al. Altered expression of MAP-2, GAP-43, and synaptophysin in the hippocampus of rats with chronic cerebral hypoperfusion correlates with cognitive impairment. Brain research Molecular brain research 2005; 139: 169-77.

22. Zhang Y, Cai GE, Yang Q, et al. Timedependent changes in learning ability and induction of long-term potentiation in the lithium-pilocarpine-induced epileptic mouse model. Epilepsy \& Behavior 2010; 17: 448-54.

23. Vorhees CV, Williams MT. Morris water maze: procedures for assessing spatial and related forms of learning and memory. Nature protocols 2006; 1: 848-58.

24. Ehara A, Ueda S. Application of Fluoro-Jade C in acute and chronic neurodegeneration models: utilities and staining differences. Acta histochemica et cytochemica 2009; 42: 171-9.

25. Ritchie LJ, De Butte M, Pappas BA. Chronic mild stress exacerbates the effects of permanent bilateral common carotid artery occlusion on CA1 neurons. Brain Research 2004; 1014: 228-35. 
